\title{
New Particles at LEP 1.5
}

\section{J.-F. Grivaz of the Laboratoire de I'Accélérateur Linéaire, IN2P3-CNRS et Université de Paris-Sud, reports on last autumn's searches for new particles at LEP using energies of 130 and $136 \mathrm{GeV}$.}

Most of the data taken at LEP, CERN's large $\mathrm{e}^{+} \mathrm{e}^{-}$collider, has used energies close to the mass, $91.2 \mathrm{GeV} / \mathrm{c}^{2}$, of the $\mathrm{Z}$ boson, with each of the four LEP experiments collecting about five million $Z$ decays into lepton pairs and into hadrons. This wealth of data has allowed precision tests of the Standard Model as well as detailed studies of the tau lepton and heavy flavoured particles. By contrast, the majority of searches for new particles were completed in a matter of weeks or months following LEP's start-up because the essential ingredient was the available centre-of-mass energy rather than the integrated luminosity. There are a few exceptions to this rule, notably the Higgs boson, the mass limit of which has steadily increased to $65 \mathrm{GeV} / \mathrm{c}^{2}$ over the years, but even here LEP had run out of steam.

A major new field of activity at LEP will open up in June 1996, when the threshold at $161 \mathrm{GeV}$ for the pair production of $\mathrm{W}$ bosons is crossed. Further upgrades of the centre-of-mass energy to $192 \mathrm{GeV}$ are foreseen. These upgrades have required the installation of a large number of superconducting radio-frequency accelerating cavities since the power dissipated by the accelerator's beams via synchrotron radiation increases with the fourth power of the energy. Sufficient cavities were available to allow a short run last November at intermediate centre-of-mass energies up to $140 \mathrm{GeV}$ (so-called LEP 1.5). Prompt commissioning combined with a good operational reliability and unexpectedly clean background conditions made the run a success, with close to $6 \mathrm{pb}^{-1} \mathrm{col}-$ lected by all four LEP experiments, shared equally between 130 and $136 \mathrm{GeV}$.

Interesting tests of the standard model in its electroweak [1] and QCD [2] sectors were performed using the data samples. No anomalies that might have arisen were observed in the total or differential rates of the various standard processes investigated. An excess in the $\mu^{+} \mu^{-} \mathrm{q} \bar{q}$ channel was reported, with five events observed instead of the 0.6 expected [3], but these events did not show other unexpected behaviour. A number of searches for various new particle species, such as excited or sequential leptons [4], were attempted; none was successful, and improved con- straints on the particle masses and couplings extending up to the new kinematic limit have been set.

It was undoubtedly the search for supersymmetric particles [5] which initially drew most of the LEP community's attention. This was motivated by the possibility that the so-called $R_{\mathrm{b}}$ anomaly (an excess in the rate of $Z$ decays into particles carrying beauty) could be explained by a contribution from supersymmetric particles, principally charginos, with masses only slightly beyond the previous LEP reach (charginos are particles similar to the $\mathrm{W}$ bosons, except that they carry half-integer spins). Owing to $R$-parity, namely the conservation of a quantum number carried by the supersymmetric particles, charginos are produced in pairs and lead, at the end of their decay chain, to the lightest supersymmetric particle (LSP) which is stable. The LSP is neutral and interacts only weakly with ordinary matter; as a consequence, the characteristic signature of supersymmetry is an apparent deficit of energy in the final state, the missing energy being carried away by the LSPs. Depending on the types of supersymmetric particle produced and their decay modes, the visible products in the final state consist of a lepton pair, a lepton accompanied by hadronic jets, or hadronic jets. All of these event topologies were analyzed by the four LEP experiments. No candidate events were selected, a result which was compatible with the low level of expected background and gave rise to improved limits on the masses of the various supersymmetric particles (scalar leptons, scalar tops, charginos - up to 68 $\mathrm{GeV} / c^{2}$ - neutralinos). Combining these findings with those previously obtained from $\mathrm{Z}$ decays, a lower limit of $13 \mathrm{GeV} / c^{2}$ can be inferred for the mass of the lightest neutralino, commonly assumed to be the LSP and one of the most popular dark matter candidates.

Rather unexpectedly, it was in the four-jet final state that an indication for new physics may have been observed. This topology was investigated in searching for the pair production of new particles decaying into hadron jets, such as excited quarks or Higgs bosons, either charged or neutral. For the latter, two different Higgs

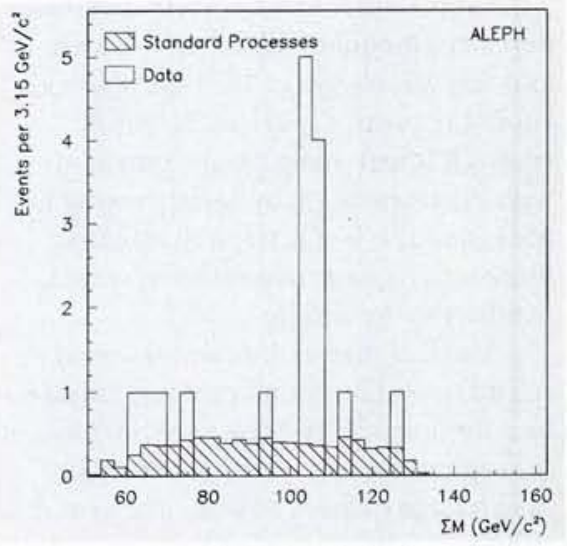

The di-jet mass sum distribution for the four-jet events selected by LEP's ALEPH collaboration. The hatched histogram gives the Standard Model expectation. Such events may represe t evidence for the pair production of new types of particles that decay into hadronic jets.

bosons would be produced in association, with masses that are possibly, but not necessarily, equal. The resolution on the di-jet mass is greatly improved by rescaling the four measured jet energies, taking into account the initial $\mathrm{e}^{+} \mathrm{e}^{-}$energy. Furthermore, using this procedure, the resolution on the sum of the two di-jet masses in an event is significantly greater than for each individual di-jet system, at least close to the kinematic limit (the smallest di-jet mass difference is chosen to extract the jet-pairing ambiguities). For di-jet mass sums larger than $90 \mathrm{GeV}$, LEP's previous reach, the ALEPH Collaboration selected 12 four-jet events while 4.8 were expected. The di-jet mass sum of nine of these events clusters within $\pm 3.2 \mathrm{GeV} / c^{2}$ of $105 \mathrm{GeV} / c^{2}$ with a resolution of $1.6 \mathrm{GeV} / c^{2}$ (see figure). Moreover, the kinematic properties of the events and the electric charge flow of their jets are not compatible with QCD expectations. However, none of the most obvious physics processes leading to four-jet final states seems able to explain all of the properties exhibited by the events. It should also be noted that the three other experiments did not see a peak in the di-jet mass sum, although, at the present level of statistics, this is essentially compatible with the ALEPH result. Deciding whether these observations result from a conspiracy of unlikely statistical fluctuations or from the pair production of some new particles decaying into hadronic jets will be settled once sufficient data has been collected.

\section{References}

[1] CERN-PPE/96-45; /95-191; /96-25.

[2] CERN-PPE/96-43; /96-05; /95-192.

[3] CERN-PPE/96-31.

[4] CERN-PPE/95-190; /96-38.

[5] CERN-PPE/96-10;/96-29;/96-20. 